Document downloaded from:

http://hdl.handle.net/10251/55414

This paper must be cited as:

Marini, S.; Soto Pacheco, P.; Mattes, M.; Gimeno Martinez, B.; Bleda Pérez, S.; Vidal Pantaleoni, A.; Boria Esbert, VE. (2014). Rigorous evaluation of propagation losses in arbitrarily shaped waveguide structures using boundary integral resonant mode expansion and perturbation of boundary conditions. IET Microwaves Antennas and Propagation. 8(12):980-989. doi:10.1049/iet-map.2013.0414.

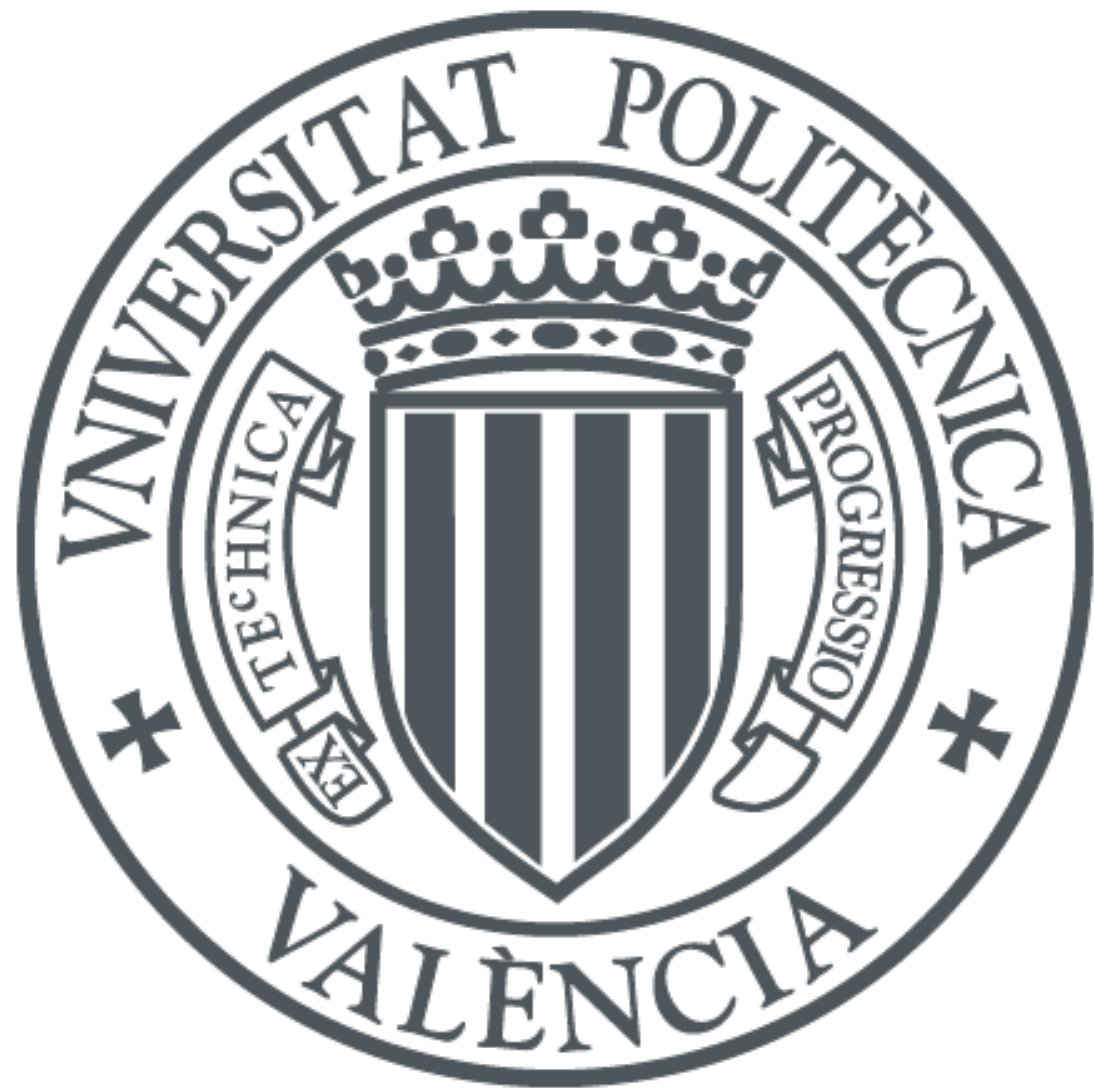

The final publication is available at

http://dx.doi.org/10.1049/iet-map.2013.0414

Copyright Institution of Engineering and Technology (IET)

Additional Information

This paper is a preprint of a paper submitted to IET Microwaves Antennas and Propagation and is subject to Institution of Engineering and Technology Copyright. If accepted, the copy of record will be available at IET Digital Library 


\title{
Rigorous Evaluation of Propagation Losses in Arbitrarily Shaped Waveguide Structures Using BI-RME and Perturbation of Boundary Conditions
}

\author{
Stephan Marini ${ }^{1}$, Pablo Soto ${ }^{2}$, Michael Mattes ${ }^{3}$, Benito Gimeno ${ }^{4}$, Sergio Bleda $^{1}$, Ana Vidal ${ }^{2}$ and Vicente \\ E. Boria $^{2}$ \\ ${ }^{1}$ Departamento de Física, Ingeniería de Sistemas y Teoría de la Señal, Universidad de Alicante, E-03690 \\ Alicante, Spain (smarini@ua.es,sergio.bleda@ua.es). \\ ${ }^{2}$ Departamento de Comunicaciones, Instituto de Telecomunicaciones y Aplicaciones Multimedia, \\ Universidad Politécnica de Valencia, E-46022, Valencia, Spain (pabsopac@dcom.upv.es, \\ avidal@dcom.upv.es,vboria@dcom.upv.es). \\ ${ }^{3}$ Laboratory of Electromagnetics and Acoustics, École Polytechnique Fédérale de Lausanne (EPFL), \\ Lausanne, Switzerland. (michael.mattes@epfl.ch). \\ ${ }^{4}$ Departamento de Física Aplicada, Instituto de Ciencia de Materiales, Universidad de Valencia, \\ E-46100, Burjassot, Spain (benito.gimeno@uv.es).
}

\begin{abstract}
The accurate consideration of propagation losses in arbitrarily shaped waveguide based structures is studied in this paper. For such a purpose, a software tool based on the perturbation of the boundary conditions on the waveguide metallic walls and on the Boundary Integral - Resonant Mode Expansion (BI-RME) method has been developed. To show the advantages of the proposed technique with respect to the classic power-loss method, the complex propagation wavenumbers of a double ridge and an elliptical waveguide have been first computed and compared with results of a commercial software based on the finite element technique. Next a circular, a sectorial shaped and a triangular shaped waveguide have been considered. Then, a computer-aided design (CAD) software package based on this modal analysis tool has been applied to predict the propagation loss effects in complex waveguide structures, such as an evanescent mode ridge waveguide filter, a traditional dual mode filter with circular cavities, and a twist component for K-band applications.
\end{abstract}

\section{INTRODUCTION}

The increasing capacity demands in telecommunication links can be fulfilled using higher frequency bands. Many applications have therefore been raised to microwave and millimeter-wave bands. For instance, the upcoming next satellite series is going to use Ka-band links to provide higher bandwidths [1]- [4]. Furthermore, future high-speed telecommunications and video streaming will work around $60 \mathrm{GHz}$, whereas automotive radars will operate around $77 \mathrm{GHz}$ [5]- [7]. At such higher frequencies, loss effects are more pronounced and can even alter the frequency response of waveguide devices. In this context, the modelling of losses becomes more and more important in 
the design of key hardware for wireless and space communication systems, such as filters, couplers, polarizers, orthomode transducers, diplexers and multiplexers [8]. As a consequence, the accurate prediction of propagation loss effects within modern computer-aided design (CAD) tools of passive microwave and millimeter-wave waveguide components is required [9]- [13].

Ohmic losses of hollow metallic waveguides are generally computed by means of the classic power loss method [14]. Applications of this classical method can be found in [15] for rectangular and circular ridge waveguides, in [16] for circular waveguides and in [17] for substrate-integrated waveguides (SIW). Such a technique provides a correction in the real part of the propagation constant, which is obtained by assuming ideal fields in the waveguide cross-section, and by imposing the Leontovich or impedance boundary condition over the contour [18]. This approach has some inconveniences: it gives meaningless results at the modal cut-off frequency, where the modal attenuation constant tends to infinity, and does not take into account the losses associated with evanescent modes.

These drawbacks can be overcome using the so-called perturbation of boundary conditions technique [19]. As recently demonstrated in [20], this method can be used in the frequency range very close to and below cut-off. Moreover, the effect of metal losses on the phase constant is also taken into account, since a complex propagation wavenumber $k_{z}=\beta-j \alpha$ for both propagative and evanescent modes is computed. Few contributions based on this method can be found in the literature. In [21] and [22] it has been used to compute the complex propagation constant of rectangular waveguide modes, whereas [23] and [24] are focused on circular and coaxial waveguide modes, respectively. However, this technique has not yet been used to model wall ohmic losses in waveguides with non-canonical shapes.

In this paper we extend the perturbation of boundary condition method to arbitrarily shaped waveguides whose contour is composed of straight, circular and elliptical arcs. The complete modal chart of such waveguides has been accurately computed using the Boundary Integral - Resonant Mode Expansion (BI-RME) method [25], [26], and the resulting modal field representation has been employed to derive the complex propagation constant of waveguide modes. This technique has been integrated into a CAD software package based on the Integral Equation (IE) technique described in [27], [28]. Finally, this CAD software package has been used to accurately predict the propagation loss effects in three different complex passive waveguide devices, providing a dramatic reduction in CPU time over current finite-element commercial simulators.

\section{Complex Modal Propagation Constant}

Let us consider a hollow waveguide with arbitrary cross-section $S$ of contour $\partial S$ (see Fig. 1). From now and on, with the aim of simplifying the notation, a zero subscript will only be used to denote the electromagnetic fields for perfectly conducting walls. Note that the time factor $e^{j \omega t}$ is considered and omitted throughout this paper, being $j$ the imaginary unit and $f=\omega /(2 \pi)$ the frequency. 


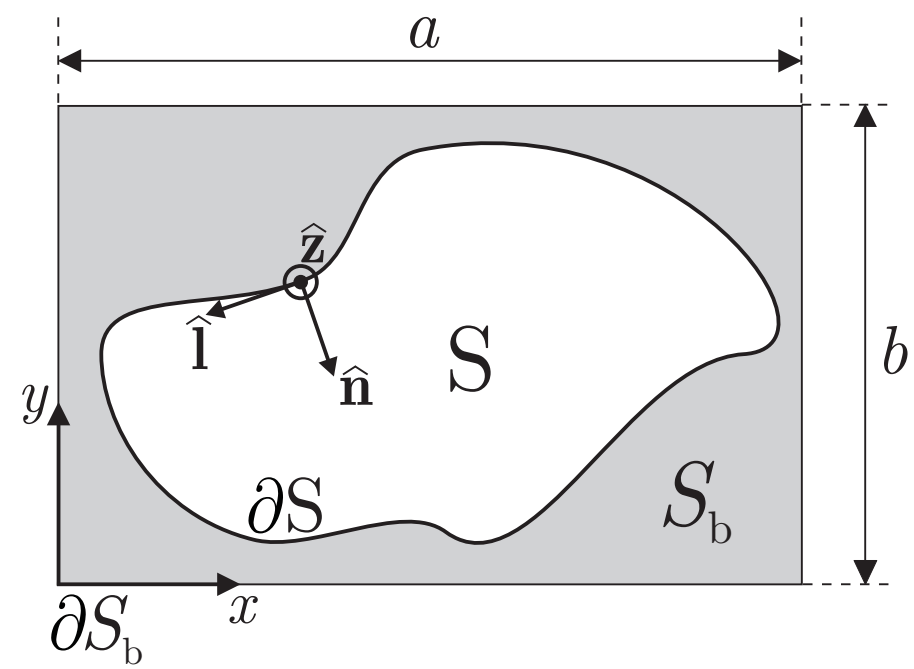

Fig. 1. Arbitrarily shaped waveguide of cross-section $S$ and contour $\partial S$ completely enclosed inside a rectangular box of cross-section $S_{b}$. Definition of variables and local coordinate system.

The complex modal propagation wavenumbers $\left(k_{z}=\beta-j \alpha\right)$ of such waveguide will be obtained from the solution of equivalent perturbed problems for both TM and TE modes, which are derived using the perturbation of boundary conditions technique. This section summarizes the main results obtained from applying such a technique, which are expressed, in contrast to [20], in a suitable form for the application of the BI-RME method as described in Section III.

\section{A. TM modes}

Following the method presented in [19], [20], the equivalent perturbed TM problem considering losses is

$$
\begin{array}{r}
\left(\nabla_{t}^{2}+k_{c}^{2}\right) \Psi=0 \\
\left.\left.\Psi\right|_{\partial S} \simeq \frac{(1-j)}{2} \delta\left(\frac{\omega}{\omega_{c 0}}\right)^{2} \frac{\partial \Psi_{0}}{\partial n}\right|_{\partial S}
\end{array}
$$

being $\Psi$ the TM scalar potential, $k_{c}$ the complex cut-off wavenumber, $\delta=\sqrt{2 / \omega \mu_{0} \sigma}$ the skin depth, $\sigma$ the electrical conductivity, and $\omega_{c 0}$ the cut-off angular frequency of the unperturbed mode (lossless case).

The new complex propagation wavenumber can be obtained after applying Green's theorem in two dimensions [19]:

$$
k_{z}^{2} \simeq k_{z 0}^{2}+\frac{(1-j)}{2} \delta\left(\frac{\omega}{\omega_{c 0}}\right)^{2} \frac{\oint_{\partial S}\left|\frac{\partial \Psi_{0}}{\partial n}\right|^{2} d l}{\int_{S}\left|\Psi_{0}\right|^{2} d S} .
$$


Furthermore, equation (2) can be simplified considering that

$$
\begin{aligned}
\mathbf{e}_{t 0} & =-\nabla_{t} \Psi_{0} \\
\mathbf{h}_{t 0} & =\widehat{\mathbf{z}} \times \mathbf{e}_{t 0} \\
\frac{\partial \Psi_{0}}{\partial n} & =\nabla_{t} \Psi_{0} \cdot \widehat{\mathbf{n}}=\left(\widehat{\mathbf{z}} \times \mathbf{h}_{t 0}\right) \cdot \widehat{\mathbf{n}}=\mathbf{h}_{t 0} \cdot \widehat{\mathbf{l}}
\end{aligned}
$$

where $\widehat{\mathbf{n}}$ is the unit normal vector inward from the conductor and $\widehat{\mathbf{l}}$ is the unit vector tangential to the conductor surface (see Fig. 1), whereas $\mathbf{e}_{t 0}$ and $\mathbf{h}_{t 0}$ are the normalized transverse electric and magnetic fields, respectively,

$$
\int_{S}\left\|\mathbf{e}_{t 0}\right\|^{2} d S=\int_{S}\left\|\mathbf{h}_{t 0}\right\|^{2} d S=\int_{S}\left\|\nabla_{t} \Psi_{0}\right\|^{2} d S=1
$$

This normalization condition can be used for the evaluation of the denominator integral in (2). From Green's first identity we obtain

$$
\begin{aligned}
\int_{S}\left\|\nabla_{t} \Psi_{0}\right\|^{2} d S & =\int_{S}\left(\nabla_{t} \Psi_{0}\right) \cdot\left(\nabla_{t} \Psi_{0}\right) d S \\
& =k_{c 0}^{2} \int_{S}\left|\Psi_{0}\right|^{2} d S=1
\end{aligned}
$$

thus from the last expression on the right-hand side of (5), we can get

$$
\int_{S}\left|\Psi_{0}\right|^{2} d S=1 / k_{c 0}^{2}
$$

As a result, and after using (3) and (6) in (2), we reach the final expression for the TM complex propagation wavenumber in an arbitrarily shaped waveguide

$$
\begin{gathered}
k_{z}^{2} \simeq k_{z 0}^{2}+\frac{(1-j)}{2} \delta\left(\frac{\omega}{\omega_{c 0}}\right)^{2} k_{c 0}^{2} \oint_{\partial S}\left|\mathbf{h}_{t 0} \cdot \widehat{\mathbf{l}}\right|^{2} d l= \\
k_{z 0}^{2}+\frac{(1-j)}{2} \delta\left(\frac{\omega}{c}\right)^{2} \oint_{\partial S}\left|\mathbf{h}_{t 0} \cdot \widehat{\mathbf{l}}\right|^{2} d l
\end{gathered}
$$

where we have used that $k_{c 0}=\omega_{c 0} / c$, being $c$ the light speed in vacuum.

\section{B. TE modes}

In a similar way, the equivalent TE eigenvalue problem considering losses is given by [20]

$$
\begin{gathered}
\left(\nabla_{t}^{2}+k_{c}^{2}\right) \Phi=0 \\
\left.\frac{\partial \Phi}{\partial n}\right|_{\partial S} \simeq-\left.\frac{(1-j)}{2} \delta\left(\omega^{2} \mu_{0} \epsilon_{0} \Phi_{0}-\frac{k_{z 0}^{2}}{k_{c 0}^{2}} \frac{\partial^{2} \Phi_{0}}{\partial l^{2}}\right)\right|_{\partial S}
\end{gathered}
$$

where $\Phi$ is the TE scalar potential and $k_{z 0}^{2}=\omega^{2} \mu_{0} \epsilon_{0}-k_{c 0}^{2}$.

Then, after using the Green theorem in two dimensions, the complex propagation wavenumber for TE modes 
can be computed by

$$
k_{z}^{2} \simeq k_{z 0}^{2}+\frac{(1-j)}{2} \delta \frac{\oint_{\partial S}\left(\omega^{2} \mu_{0} \epsilon_{0}\left|\Phi_{0}\right|^{2}+\frac{k_{z 0}^{2}}{k_{c 0}^{2}}\left|\frac{\partial \Phi_{0}}{\partial l}\right|^{2}\right) d l}{\int_{s}\left|\Phi_{0}\right|^{2} d S} .
$$

As it was done for TM modes, this equation can be simplified considering that

$$
\begin{aligned}
\mathbf{e}_{t 0} & =\widehat{\mathbf{z}} \times \nabla_{t} \Phi_{0} \\
\mathbf{h}_{t 0} & =\widehat{\mathbf{z}} \times \mathbf{e}_{t 0}=-\nabla_{t} \Phi_{0} \\
\frac{\partial \Phi_{0}}{\partial l} & =\nabla_{t} \Phi_{0} \cdot \widehat{\mathbf{l}}=-\mathbf{h}_{t 0} \cdot \widehat{\mathbf{l}}
\end{aligned}
$$

and also the relationship between $h_{z 0}$ and $\Phi_{0}$

$$
h_{z 0}=\frac{k_{c 0}^{2}}{k_{z 0}} \Phi_{0}
$$

Since the TE scalar potential is also normalized according to $\int_{S}\left\|\nabla_{t} \Phi_{0}\right\|^{2} d S=1$, from Green's first identity (5) it follows that

$$
\int_{S}\left|\Phi_{0}\right|^{2} d S=1 / k_{c 0}^{2}
$$

Finally, after using (10), (11) and (12) in (9), the corresponding complex propagation wavenumber for TE modes in an arbitrarily shaped waveguide can be written as

$$
\begin{aligned}
k_{z}^{2} \simeq & k_{z 0}^{2}+\frac{(1-j)}{2} \delta k_{z 0}^{2} \oint_{\partial S}\left(\frac{\omega^{2} \mu_{0} \epsilon_{0}}{k_{c 0}^{2}}\left|h_{z 0}\right|^{2}+\left|\mathbf{h}_{t 0} \cdot \widehat{\mathbf{l}}\right|^{2}\right) d l \\
& =k_{z 0}^{2}+\frac{(1-j)}{2} \delta k_{z 0}^{2}\left[\left(\frac{\omega}{\omega_{c 0}}\right)^{2} \oint_{\partial S}\left|h_{z 0}\right|^{2} d l\right. \\
& \left.+\oint_{\partial S}\left|\mathbf{h}_{t 0} \cdot \widehat{\mathbf{l}}\right|^{2} d l\right]
\end{aligned}
$$

Note that in both (7) and (13) the dependence with $\omega$ is extracted from the integrals, which allows to compute them using a suitable numerical algorithm only once for each involved mode.

\section{ApPlicAtion OF THE BI-RME METHOD}

\section{A. Computation of modal spectrum and magnetic fields}

To evaluate (7) and (13), we need to know the modal chart of the involved arbitrary shaped waveguide, as well as the magnetic fields of its modes on the contour $\partial S$. To reach this aim, the well-known BI-RME method has been used [25], and more specifically the extension proposed in [26]. This particular implementation allows a rigorous treatment of waveguides whose arbitrarily shaped contour $\partial S$ is composed of any arbitrary combination of straight, circular and elliptical arcs. 
The transverse magnetic field of a TM and a TE mode of the arbitrary shaped waveguide is given by [26]

$$
\begin{aligned}
\mathbf{h}_{t 0}^{T M}(\mathbf{r})= & \widehat{\mathbf{z}} \times\left[-\sum_{n} b_{n}^{\prime} \oint_{\partial S} \nabla_{t} g\left(\mathbf{r}, \mathbf{s}^{\prime}\right) u_{n}\left(l^{\prime}\right) d l^{\prime}\right. \\
& \left.+\sum_{m} \frac{a_{m}^{\prime}}{\tilde{k}_{m}^{\prime}} \tilde{\mathbf{e}}_{m}^{T M}(\mathbf{r})\right] \\
\mathbf{h}_{t 0}^{T E}(\mathbf{r})=\hat{\mathbf{z}} \times & {\left[\frac{1}{k_{c 0}} \sum_{n} b_{n} \oint_{\partial S} \nabla_{t} g\left(\mathbf{r}, \mathbf{s}^{\prime}\right) \frac{\partial w_{n}\left(l^{\prime}\right)}{\partial l^{\prime}} d l^{\prime}\right.} \\
& +k_{c 0}\left(\sum_{n} b_{n} \oint_{\partial S} \overline{\mathbf{G}}_{s t}\left(\mathbf{r}, \mathbf{s}^{\prime}\right) \cdot \hat{\mathbf{l}}\left(l^{\prime}\right) w_{n}\left(l^{\prime}\right) d l^{\prime}\right. \\
& \left.\left.+\sum_{m} \frac{\tilde{\mathbf{e}}_{m}^{T E}(\mathbf{r})}{\tilde{k}_{m}^{2}} a_{m}\right)\right]
\end{aligned}
$$

where $\mathbf{r}(x, y)$ and $\mathbf{s}^{\prime}\left(x^{\prime}, y^{\prime}\right)$ are generic observation and source points, respectively; $g$ is the static 2-D Green's function (satisfying Dirichlet boundary conditions) for the rectangular box $S_{b}$ (see Fig. 1); $\overline{\mathbf{G}}_{s t}$ is a rapidly convergent expression of the solenoidal dyadic Green's function of such a rectangular resonator [25]; $\tilde{\mathbf{e}}_{m}^{T M}, \tilde{k}_{m}^{\prime}, \tilde{\mathbf{e}}_{m}^{T E}$, and $\tilde{k}_{m}$ are the normalized modal vectors and cut-off wavenumbers of the TM and TE rectangular resonator modes, respectively; and $a_{m}^{\prime}, a_{m}, b_{n}^{\prime}$ and $b_{n}$ are the modal expansion coefficients $\left(a_{m}^{\prime}, a_{m}\right)$ and amplitudes of the longitudinal $\left(b_{n}^{\prime}\right)$ and transverse $\left(b_{n}\right)$ components of the current density on the arbitrary contour at cut-off. The last four parameters and the cut-off wavenumber $k_{c 0}$ of each arbitrary waveguide mode are obtained after solving an integral equation via the Galerkin version of the Method of Moments, where the basis functions $u_{n}$ (TM case) and $w_{n}$ (TE case) are chosen to be overlapping piecewise parabolic splines, as detailed in [26].

Finally, and according to (13), the evaluation of the propagation constant of a TE mode also requires to know the magnitude of the axial component of the magnetic field $h_{z 0}^{T E}$ on the contour $\partial S$, which can be easily derived from the transverse surface current density at cut-off

$$
\left|h_{z 0}^{T E}(\mathbf{r})\right|_{\partial S}=\left\|\widehat{\mathbf{n}} \times \mathbf{J}_{t}(l)\right\|=\left|\sum_{n} b_{n} w_{n}(l)\right| .
$$

Observe that all the modal terms included in the expressions (7) and (13) used to compute the complex propagation constant can be derived from the modal information provided by the BI-RME method.

\section{B. Solving the contour integrals}

The numerical evaluation of expressions (7) and (13) implies the computation of the modal magnetic field at certain boundary points, which is carried out by means of (14), (15) and (16). To evaluate the fields, the integrals in (14) and (15) are normally split into different segments (basic contour element of the BI-RME method) supporting each basis function $u_{n}$ or $w_{n}$. Each subintegral is then computed in a numerical way following a simple 
Gauss-Legendre quadrature rule. However, this numerical procedure cannot be applied when the observation point $\mathbf{r}(x, y)$ approaches a source point $\mathbf{s}^{\prime}\left(x^{\prime}, y^{\prime}\right)$ on the contour $\partial S$ of the arbitrary waveguide, since singular integrals (corresponding to the singularities of the $g$ and $\overline{\mathbf{G}}_{s t}$ terms) appear. This situation occurs whenever the observation point belongs to the particular segment where the integration is carried out.

Taking into account that the dyadic Green's function $\overline{\mathbf{G}}_{s t}$ behaves as $\log \left|\mathbf{r}-\mathbf{s}^{\prime}\right|^{2}$ when $\mathbf{s}^{\prime} \rightarrow \mathbf{r}$, the second integral in (15) can be evaluated following the procedure (with minor modifications) already described in [26], and will not be discussed here. However, in (14) and (15) appear two new types of integrals that deserve a detailed description. These integrals are related to the transverse gradient of the static 2D Green's function

$$
\nabla_{t} g=\frac{\partial g}{\partial x} \widehat{\mathbf{x}}+\frac{\partial g}{\partial y} \widehat{\mathbf{y}}
$$

which has the following rapidly convergent expression due to the Poisson summation formula:

$$
\begin{aligned}
& \frac{\partial g}{\partial x}=\frac{1}{4 a} \sum_{s=-\infty}^{\infty} C_{s}^{10}+C_{s}^{01}-C_{s}^{00}-C_{s}^{11} \\
& \frac{\partial g}{\partial y}=\frac{1}{4 b} \sum_{r=-\infty}^{\infty} K_{r}^{10}+K_{r}^{01}-K_{r}^{00}-K_{r}^{11}
\end{aligned}
$$

with

$$
\begin{aligned}
C_{s}^{p q} & =\frac{\sin \left(T_{p}^{x}\right)}{\cosh \left[\frac{\pi}{a}\left(y-(-1)^{q} y^{\prime}+2 s b\right)\right]-\cos \left(T_{p}^{x}\right)} \\
K_{r}^{p q} & =\frac{\sin \left(T_{p}^{y}\right)}{\cosh \left[\frac{\pi}{b}\left(x-(-1)^{q} x^{\prime}+2 r a\right)\right]-\cos \left(T_{p}^{y}\right)}
\end{aligned}
$$

where $T_{p}^{x}=\frac{\pi}{a}\left(x-(-1)^{p} x^{\prime}\right)$ and $T_{p}^{y}=\frac{\pi}{b}\left(y-(-1)^{p} y^{\prime}\right)$.

The singularity lies on $C_{0}^{00}$ and $K_{0}^{00}$, which are the only terms that tend to infinity when the field point approaches the source point. In this case, the gradient of the static 2D Green's function (see (17)-(19)) can be split into a singular part $\nabla_{t} g_{s}$ including the singular terms

$$
\nabla_{t} g_{s}=\frac{\partial g_{s}}{\partial x} \widehat{\mathbf{x}}+\frac{\partial g_{s}}{\partial y} \widehat{\mathbf{x}}=-\left[\frac{C_{0}^{00}}{4 a} \widehat{\mathbf{x}}+\frac{K_{0}^{00}}{4 b} \widehat{\mathbf{y}}\right]
$$

so that

$$
\begin{aligned}
\frac{\partial g_{s}}{\partial x} & =-\frac{1}{4 a} \frac{\sin \left(\frac{\pi}{a}\left(x-x^{\prime}\right)\right)}{\cosh \left(\frac{\pi}{a}\left(y-y^{\prime}\right)\right)-\cos \left(\frac{\pi}{a}\left(x-x^{\prime}\right)\right)} \\
\frac{\partial g_{s}}{\partial y} & =-\frac{1}{4 b} \frac{\sin \left(\frac{\pi}{b}\left(y-y^{\prime}\right)\right)}{\cosh \left(\frac{\pi}{b}\left(x-x^{\prime}\right)\right)-\cos \left(\frac{\pi}{b}\left(y-y^{\prime}\right)\right)}
\end{aligned}
$$

and a regular part $\nabla_{t} g_{r}$ containing the remaining terms in (18). The singular term can be divided again into two new components, a term $\nabla_{T} g_{s s}$ isolating the singularity (of the form $1 /\left|\mathbf{r}-\mathbf{s}^{\prime}\right|$ ) and a regular term $\nabla_{T} g_{s r}=$ 
$\nabla_{t} g_{s}-\nabla_{T} g_{s s}$ that tends to zero when $\mathbf{s}^{\prime} \rightarrow \mathbf{r}$. The particular expression for both terms depends on the contour shape (i.e. straight, circular or elliptical).

Using the same parametrization of straight, circular and elliptical shapes already employed in [26], with $\xi$ being the dummy normalized parameter between $[-0.5,0.5]$ that runs the segments composing each arc, it can be proved that for a straight segment of length $\mathrm{L}$ and angle $\theta$ (defined between the linear arc and the $x$-axis) whose points are given by

$$
\begin{aligned}
& x=x_{0}+(\xi+0.5) L \cos \theta \\
& y=y_{0}+(\xi+0.5) L \sin \theta
\end{aligned}
$$

where $\left(x_{0}, y_{0}\right)$ is the coordinate of the starting segment point, the singular term $\nabla_{t} g_{s s}$ is of the form

$$
\nabla_{t} g_{s s}=-\frac{\cos \theta \widehat{\mathbf{x}}+\sin \theta \widehat{\mathbf{y}}}{2 \pi L\left(\xi-\xi^{\prime}\right)}
$$

On the other hand, for a circular segment of radius $r$, center $\left(x_{0}, y_{0}\right)$, initial angle $\varphi_{1}$ and final angle $\varphi_{2}$

$$
\begin{aligned}
& x=x_{0}+r \cos \varphi(\xi) \\
& y=y_{0}+r \sin \varphi(\xi)
\end{aligned}
$$

with $\varphi(\xi)=\varphi_{1}+\Delta \varphi(\xi+0.5)$ and $\Delta \varphi=\varphi_{2}-\varphi_{1}$, the isolated singular term is

$$
\nabla_{t} g_{s s}=\frac{\sin \varphi(\xi) \widehat{\mathbf{x}}-\cos \varphi(\xi) \widehat{\mathbf{y}}}{2 \pi r \Delta \varphi\left(\xi-\xi^{\prime}\right)}
$$

Finally, for an elliptical segment with major and minor semiaxes $a_{e}$ and $b_{e}$, initial and final elliptical angles $\eta_{1}$ and $\eta_{2}$, which is rotated an angle $\alpha$ with respect to the $x$-axis, the parametrization in terms of $\xi$ takes the form

$$
\begin{aligned}
& x=x_{0}+a_{e} \cos \alpha \cos \eta(\xi)-b_{e} \sin \alpha \sin \eta(\xi) \\
& y=y_{0}+a_{e} \sin \alpha \cos \eta(\xi)+b_{e} \cos \alpha \sin \eta(\xi)
\end{aligned}
$$

being $\left(x_{0}, y_{0}\right)$ the center of the ellipse, $\eta(\xi)=\eta_{1}+\Delta \eta(\xi+0.5)$ the elliptical angle and $\Delta \eta=\eta_{2}-\eta_{1}$, the extracted singular term $\nabla_{t} g_{s s}$ is now given by

$$
\nabla_{t} g_{s s}=\frac{K_{e}^{x} \widehat{\mathbf{x}}+K_{e}^{y} \widehat{\mathbf{y}}}{2 \pi a_{e} \Delta \eta\left(\xi-\xi^{\prime}\right)}
$$


with

$$
\begin{aligned}
K_{e}^{x} & =\frac{\cos \alpha \sin \eta(\xi)+\left(b_{e} / a_{e}\right) \sin \alpha \cos \eta(\xi)}{\sin ^{2} \eta(\xi)+\left(b_{e} / a_{e}\right)^{2} \cos ^{2} \eta(\xi)} \\
K_{e}^{y} & =\frac{\sin \alpha \sin \eta(\xi)-\left(b_{e} / a_{e}\right) \cos \alpha \cos \eta(\xi)}{\sin ^{2} \eta(\xi)+\left(b_{e} / a_{e}\right)^{2} \cos ^{2} \eta(\xi)} .
\end{aligned}
$$

The singular integrals in (14) and (15), related to the transverse gradient of $g$, can now be split into a regular one with $\nabla_{t} g_{r}+\nabla_{t} g_{s r}$, that can be straightforwardly computed using a numerical algorithm, and a contribution including $\nabla_{t} g_{s s}$ that must be analytically solved.

For the TM case, the basic singular integral (using the normalized parameter $\xi^{\prime}$ ) involves the parabolic expression of the generic basis function $u_{n}$ in the segment, $c_{2} \xi^{\prime 2}+c_{1} \xi^{\prime}+c_{0}$, and the term $\nabla_{t} g_{s s}\left(\xi, \xi^{\prime}\right)$ corresponding to the particular segment shape. If a straight segment is used, this analytical integral is given by

$$
\begin{array}{r}
L \int_{-0.5}^{0.5}-\frac{\cos \theta \widehat{\mathbf{x}}+\sin \theta \widehat{\mathbf{y}}}{2 \pi L\left(\xi-\xi^{\prime}\right)}\left(c_{2} \xi^{\prime 2}+c_{1} \xi^{\prime}+c_{0}\right) d \xi^{\prime} \\
=-\frac{\cos \theta \widehat{\mathbf{x}}+\sin \theta \widehat{\mathbf{y}}}{2 \pi} I_{s}\left(\xi, c_{2}, c_{1}, c_{0}\right)
\end{array}
$$

where $I_{s}$ is the singular integral defined in Appendix A. For a circular segment, we have

$$
\begin{array}{r}
r|\Delta \varphi| \int_{-0.5}^{0.5} \frac{\sin \varphi(\xi) \widehat{\mathbf{x}}-\cos \varphi(\xi) \widehat{\mathbf{y}}}{2 \pi r \Delta \varphi\left(\xi-\xi^{\prime}\right)}\left(c_{2} \xi^{\prime 2}+c_{1} \xi^{\prime}+c_{0}\right) d \xi^{\prime} \\
= \pm \frac{\sin \varphi(\xi) \widehat{\mathbf{x}}-\cos \varphi(\xi) \widehat{\mathbf{y}}}{2 \pi} I_{s}\left(\xi, c_{2}, c_{1}, c_{0}\right)
\end{array}
$$

using the positive sign for an anticlockwise segment (i.e., if $\varphi_{2}>\varphi_{1}$ so that $\Delta \varphi>0$ ).

On the other hand, the resulting integral in the elliptical case must be solved using a more elaborated procedure since now $d l^{\prime}=f_{e}\left(\xi^{\prime}\right) d \xi^{\prime}$ with

$$
f_{e}\left(\xi^{\prime}\right)=a_{e}|\Delta \eta| \sqrt{1-e^{2} \cos ^{2}\left(\eta\left(\xi^{\prime}\right)\right)}
$$

where $e$ denotes the ellipse eccentricity. The singular integral can therefore be written as

$$
\begin{array}{r}
a_{e}|\Delta \eta| \int_{-0.5}^{0.5} \frac{K_{e}^{x} \widehat{\mathbf{x}}+K_{e}^{y} \widehat{\mathbf{y}}}{2 \pi a_{e} \Delta \eta\left(\xi-\xi^{\prime}\right)}\left(c_{2} \xi^{\prime 2}+c_{1} \xi^{\prime}+c_{0}\right) f_{e}\left(\xi^{\prime}\right) d \xi^{\prime} \\
= \pm \frac{K_{e}^{x} \widehat{\mathbf{x}}+K_{e}^{y} \widehat{\mathbf{y}}}{2 \pi} \int_{-0.5}^{0.5} \frac{c_{2} \xi^{\prime 2}+c_{1} \xi^{\prime}+c_{0}}{\xi-\xi^{\prime}} f_{e}\left(\xi^{\prime}\right) d \xi^{\prime} \\
= \pm \frac{K_{e}^{x} \widehat{\mathbf{x}}+K_{e}^{y} \widehat{\mathbf{y}}}{2 \pi} f_{e}(\xi)\left[I_{s}\left(\xi, c_{2}, c_{1}, c_{0}\right)\right. \\
\left.-\int_{-0.5}^{0.5} \frac{c_{2} \xi^{\prime 2}+c_{1} \xi^{\prime}+c_{0}}{\xi-\xi^{\prime}}\left(1-\frac{f_{e}\left(\xi^{\prime}\right)}{f_{e}(\xi)}\right) d \xi^{\prime}\right]
\end{array}
$$

because $f_{e}\left(\xi^{\prime}\right)=f_{e}(\xi)\left[1-\left(1-f_{e}\left(\xi^{\prime}\right) / f_{e}(\xi)\right)\right]$.

Again, the positive sign in (32) is used for anticlockwise segments where $\Delta \eta>0$. It is also worth saying that 
from L'Hôpital rule

$$
\left.\frac{1-f_{e}\left(\xi^{\prime}\right) / f_{e}(\xi)}{\xi-\xi^{\prime}}\right|_{\xi^{\prime}=\xi}=\frac{\Delta \eta}{2} e^{2} \frac{\sin (2 \eta(\xi))}{f_{e}^{2}(\xi)}
$$

so that the last integral in (32) can also be computed using a numerical integration procedure.

The analytical integrals corresponding to the transverse gradient of $g$ in the TE case (see first integral in (15)) can be evaluated more easily since

$$
\frac{\partial w_{n}\left(l^{\prime}\right)}{\partial l^{\prime}} d l^{\prime}=\frac{\partial w_{n}\left(l^{\prime}\right)}{\partial \xi^{\prime}} \frac{\partial \xi^{\prime}}{\partial l^{\prime}} d l^{\prime}=\left(2 d_{2} \xi^{\prime}+d_{1}\right) d \xi^{\prime}
$$

thus expressions (29) and (30) are valid if both are divided by the segment length and also $I_{s}\left(\xi, c_{2}, c_{1}, c_{0}\right)$ is replaced with $I_{s}\left(\xi, 0,2 d_{2}, d_{1}\right)$, being $d_{2}, d_{1}$ and $d_{0}$ the coefficients of the parabolic expression of the basis function $w_{n}\left(l^{\prime}\right)$ in the integration segment.

In the elliptical TE case the term $f_{e}(\xi)$ does not appear due to (34), and the singular integral is now given by

$$
\begin{array}{r}
a_{e}|\Delta \eta| \int_{-0.5}^{0.5} \frac{K_{e}^{x} \widehat{\mathbf{x}}+K_{e}^{y} \widehat{\mathbf{y}}}{2 \pi a_{e} \Delta \eta\left(\xi-\xi^{\prime}\right)}\left(2 d_{2} \xi^{\prime}+d_{1}\right) d \xi^{\prime} \\
=\frac{K_{e}^{x} \widehat{\mathbf{x}}+K_{e}^{y} \widehat{\mathbf{y}}}{2 \pi a_{e} \Delta \eta} I_{s}\left(\xi, 0,2 d_{2}, d_{1}\right)
\end{array}
$$

where $I_{s}$ is the singular integral given in Appendix A.

\section{Results}

In this section, the accuracy of the proposed technique is completely verified with several application examples. First, we have computed the complex modal propagation constants of three well-known waveguides, i.e., a double ridge waveguide, an elliptical waveguide and a circular waveguide. These results have been successfully validated with numerical data provided by the commercial software HFSS [29], and have also been compared with the classic power-loss method. Next a circular sectorial shaped and a triangular shaped waveguide have been considered. Then, we have analyzed and predicted the propagation loss effects in three complex passive waveguide devices: an evanescent mode ridge waveguide filter, a dual mode filter with circular waveguide cavities, and a twist component for K-band applications. All the simulations have been obtained on a Intel Core i7-970 platform at $3.2 \mathrm{GHz}$ with 12-GB RAM.

\section{A. Study of propagation losses in arbitrarily shaped waveguides}

First of all, to fully validate the theory developed, we have performed the modal analysis of a double ridge WR-75 $(19.05 \times 9.525 \mathrm{~mm})$ waveguide with a finite conductivity value of $\sigma=5.8 \cdot 10^{7} \mathrm{~S} / \mathrm{m}$. Figure 2(a) shows in a semi-logarithmic scale the attenuation constant for some of the first double ridge waveguide modes $(h=2.976$ $\mathrm{mm}$ and $w=4 \mathrm{~mm}$ ) computed using the power-loss method, the proposed method based on the perturbation of 


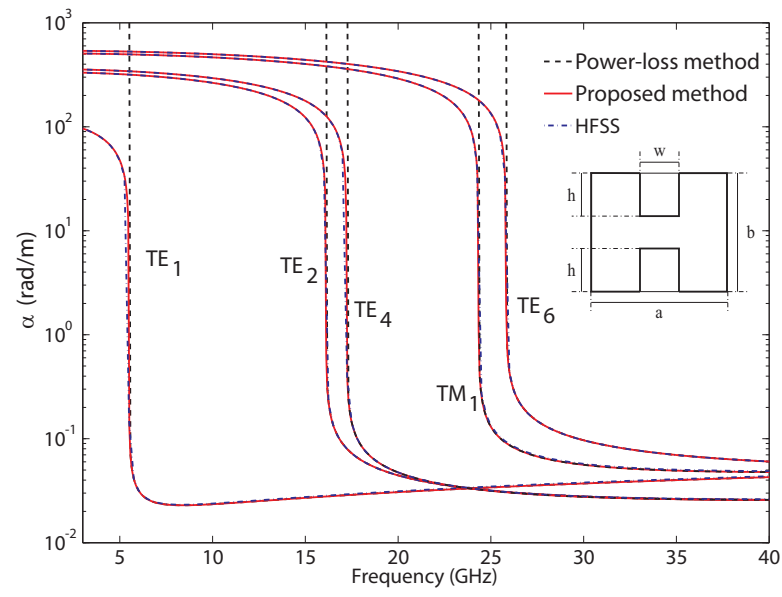

(a)

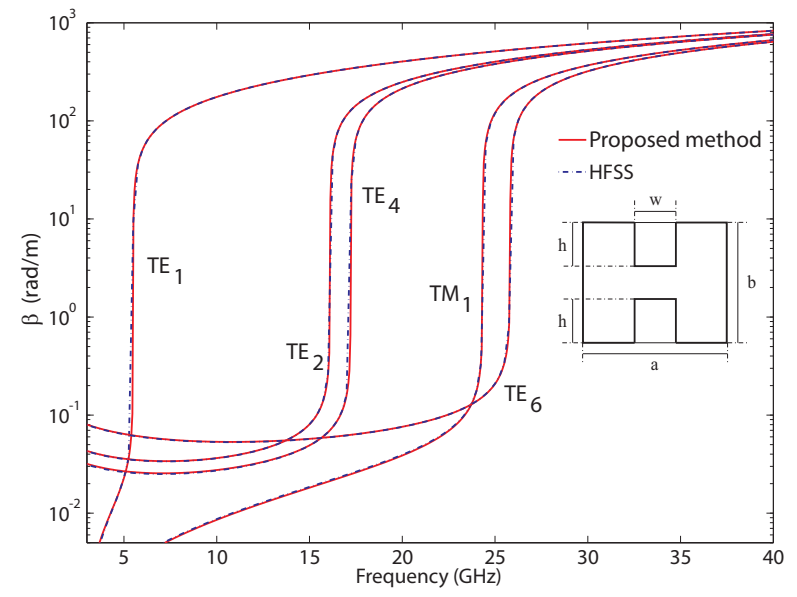

(b)

Fig. 2. Attenuation (a) and phase constant (b) for some of the first double ridge waveguide modes $(h=2.976 \mathrm{~mm}$ and $w=4 \mathrm{~mm})$ in a standard WR-75 waveguide with $\sigma=5.8 \cdot 10^{7} \mathrm{~S} / \mathrm{m}$.

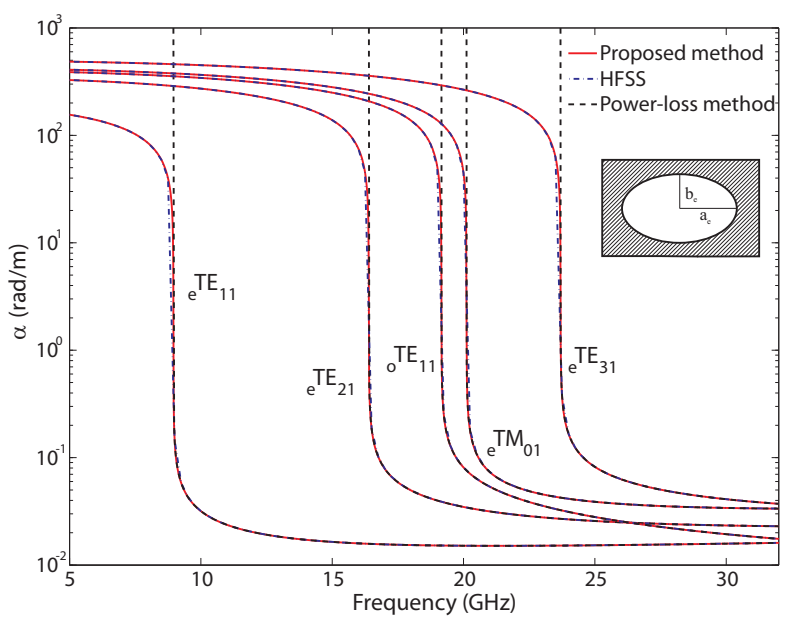

(a)

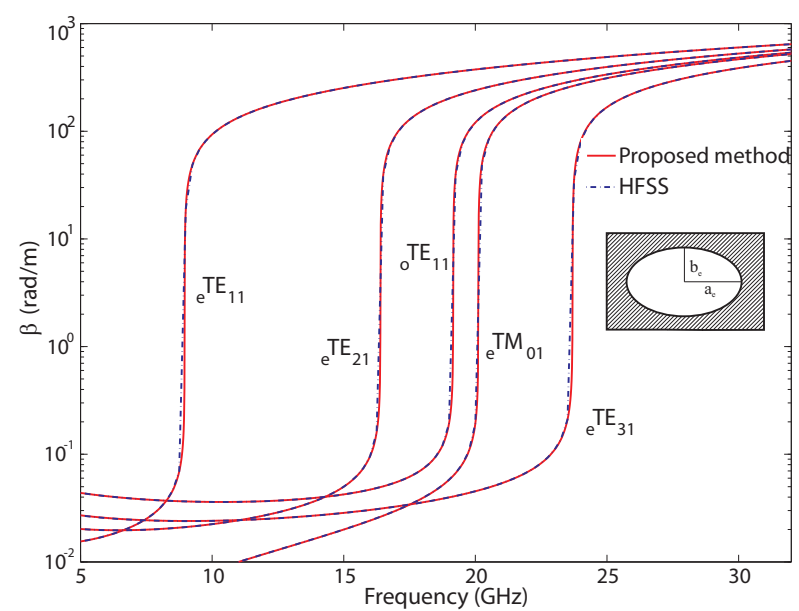

(b)

Fig. 3. Attenuation (a) and phase constant (b) for the first five elliptical waveguide modes $\left(a_{e}=10 \mathbf{m m}\right.$ and $b_{e}=4.3589$ $\mathbf{m m}$ ), with a finite conductivity value of $\sigma=5.8 \cdot 10^{7} \mathrm{~S} / \mathbf{m}$.

the boundary conditions and HFSS. Note that the proposed technique does not fail at the cut-off frequency, in contrast with the classic power-loss technique that provides an infinite value for $\alpha$. In Fig. 2(b) we also represent the phase constant for the same modes. The results predicted by the proposed technique are successfully compared with HFSS data for each analysis frequency. However, the computer time employed by the proposed technique has been $0.89 \mathrm{~s}$ per frequency point, while the computer time required by the HFSS code in the discrete sweep has been 31 s per frequency point (after using 4926 triangles in the wave port).

The next example deals with the analysis of an elliptical waveguide with major semiaxis $a_{e}=10 \mathrm{~mm}$, minor semiaxis $b_{e}=4.3589 \mathrm{~mm}$ (eccentricity $e=0.9$ ), and a finite conductivity value of $\sigma=5.8 \cdot 10^{7} \mathbf{S} / \mathbf{m}$. 


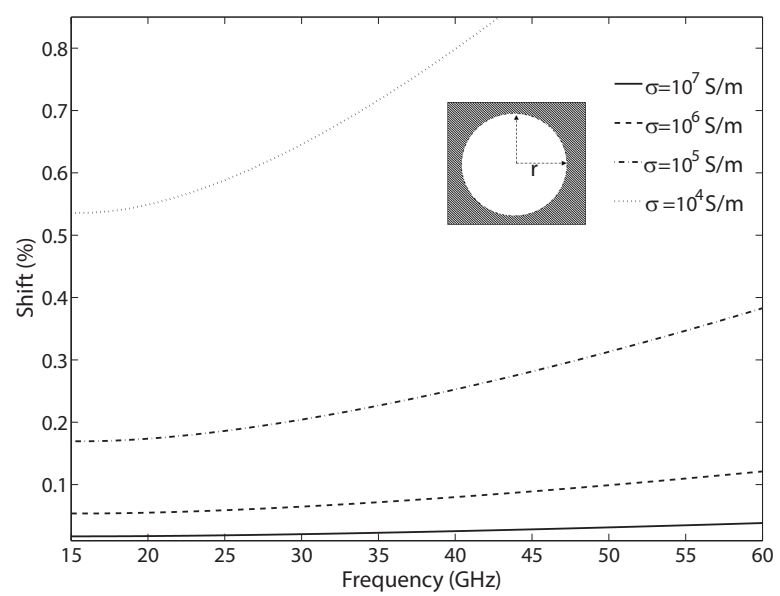

Fig. 4. Relative shift of the $T E_{11}$ cut-off wavenumber of a circular waveguide $(r=5 \mathbf{m m})$ for different conductivities.

For verification purposes, Fig. 3 compares in a semi-logarithmic scale the attenuation constant and the phase constant for the first five elliptical waveguide modes computed with the power-loss method, the proposed method and HFSS. As it can be observed, an excellent agreement between our results and HFSS data is obtained.

In order to demonstrate the variation of the cut-off wavenumber in presence of losses, Fig 4 shows the relative shift $\left(\left|\left(k_{c 0}-\operatorname{Real}\left\{k_{c}\right\}\right) / k_{c 0}\right|\right)$ for the $T E_{11}$ cut-off wavenumber of a circular waveguide $(r=5$ $\mathrm{mm}$ ) in terms of conductivity. The difference between lossless and lossy cut-off wavenumbers increases with frequency and for lower conductivity.

Once the proposed theory has been successfully validated with well-known waveguides and to demonstrate the power of the proposed technique, we have analyzed two additional arbitrarily shaped waveguides: a circular sectorial and a triangular shaped waveguide [30], [31]. In Fig. 5 the attenuation and phase constant for the first three modes of a $\mathbf{9 0}^{\circ}$ circular sectorial waveguide $(r=6 \mathbf{m m})$ with a finite conductivity value of $\sigma=10^{7} \mathrm{~S} / \mathbf{m}$ are represented. Fig. 6 plots the complex modal propagation constants for the first three triangular shaped waveguide modes $(a=7 \mathbf{m m}$ and $h=5 \mathbf{m m}$, with these dimensions the waveguide do-not have analytic solution) with the same conductivity value of $\sigma=10^{7} \mathrm{~S} / \mathrm{m}$ are alse-represented.

\section{B. Full-wave analysis of complex structures including propagation losses}

After the validation of the proposed technique with the previous benchmark tests, it is applied to the analysis of modern passive devices involving arbitrarily shaped waveguides. For such purpose, we have integrated the method presented in this paper into a CAD software package based on the integral-equation technique described in [28]. The application of this full-wave technique requires the knowledge of the modal chart of all arbitrarily shaped waveguide sections involved in the structure, which is determined following the BI-RME technique [26], as 


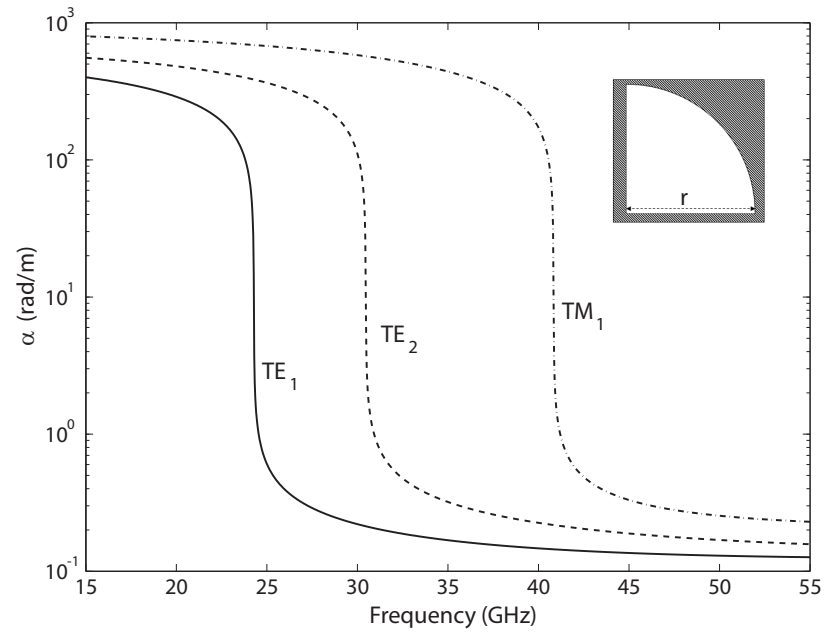

(a)

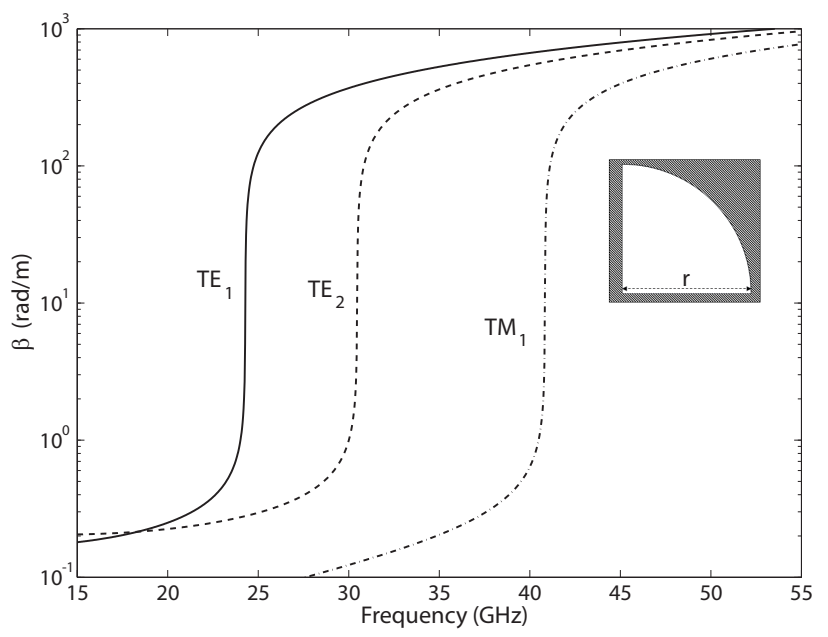

(b)

Fig. 5. Attenuation (a) and phase constant (b) for the first three circular sectorial shaped waveguide modes $(r=6$ mm), with a finite conductivity value of $\sigma=10^{7} \mathrm{~S} / \mathbf{m}$.

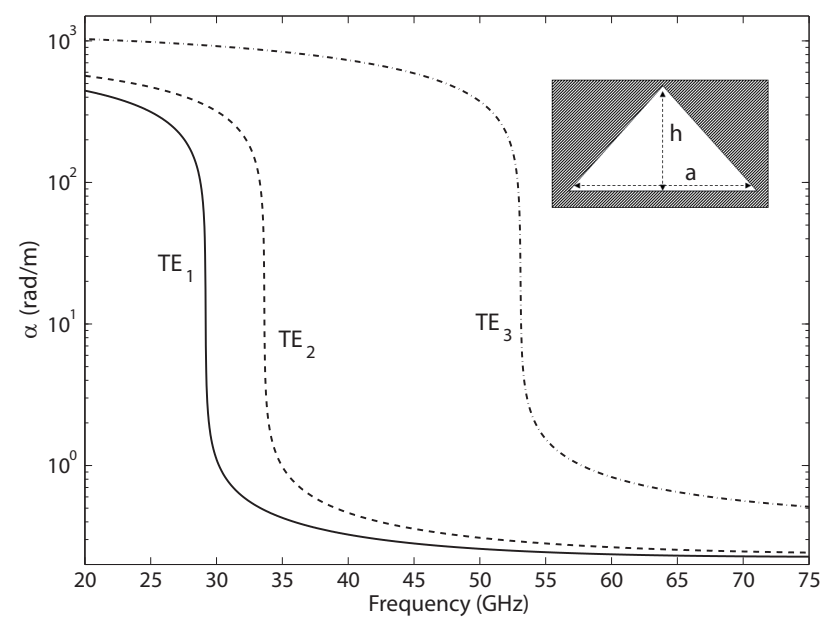

(a)

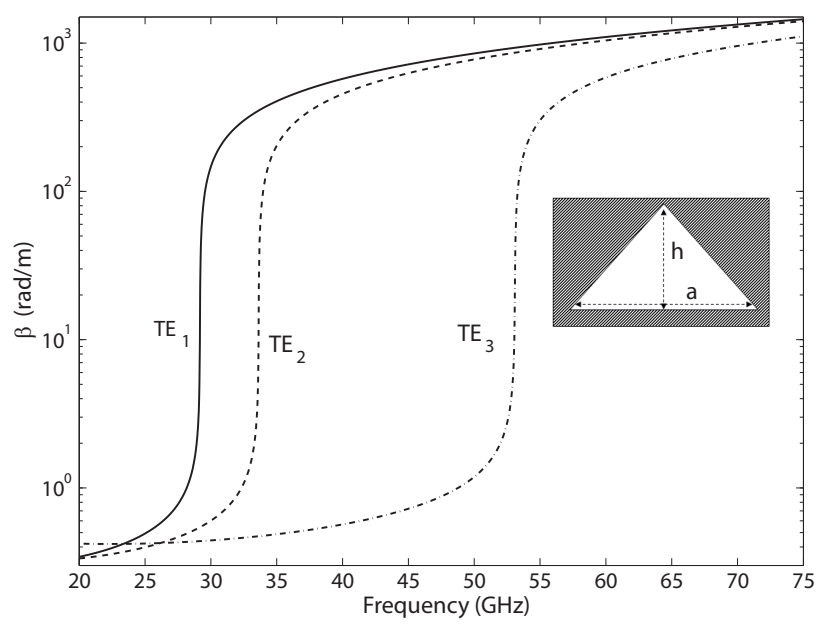

(b)

Fig. 6. Attenuation (a) and phase constant (b) for the three triangular shaped waveguide modes $(a=7 \mathbf{~ m m}$ and $h=5 \mathbf{m m})$, with a finite conductivity value of $\sigma=10^{7} \mathrm{~S} / \mathbf{m}$.

well as the new complex propagation constants computed as indicated in Section II. The final objective is to obtain a multimodal representation of each junction and each waveguide section in terms of generalised impedance matrices (GIM), which are finally connected to derive the electromagnetic response of the entire structure.

First, we have considered a five order evanescent mode waveguide filter with ridges in both the upper and lower walls, originally designed in [32]. These filters provide compact size and excellent out-of-band response, carrying out some of the design requirements for passive waveguide filters in modern communication systems for space and 


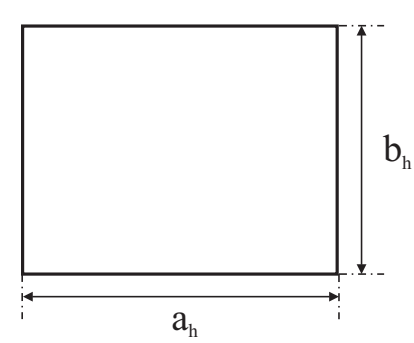

(a)

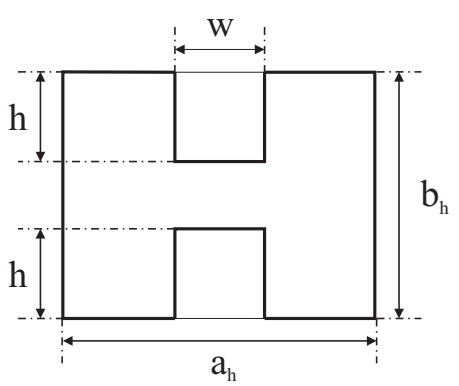

(b)

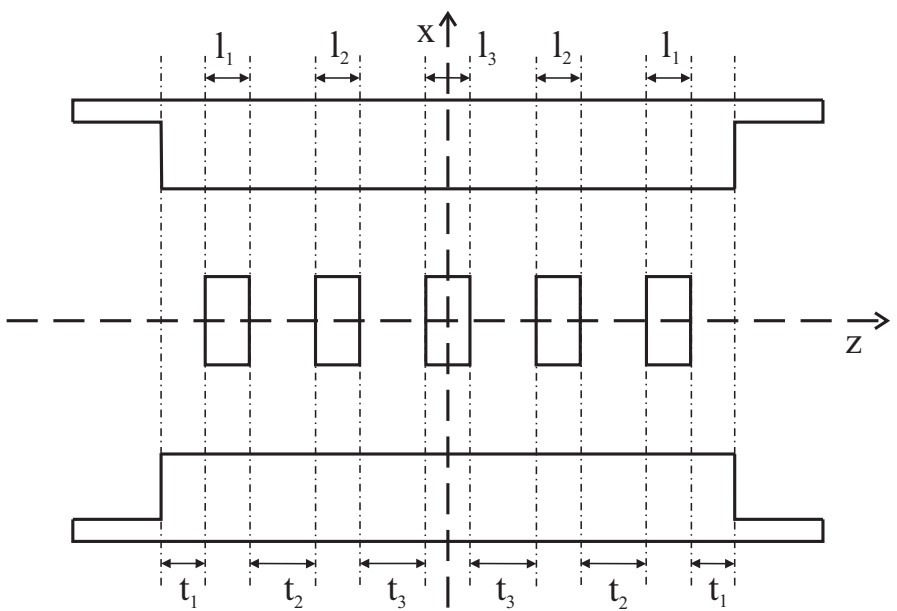

(c)

Fig. 7. Geometrical parameters of the symmetrical evanescent mode ridge waveguide filter: in a) the transverse section of the housing; in b) the transverse section of the ridge waveguides; in c) the longitudinal section of the filter.

TABLE I

DIMENSIONS (in mm) OF THE FIVE-ORDER EVANESCENT MODE RIDGE WAVEGUIDE FILTER $\left(a_{h} \times b_{h}=6.0 \times 7.49 \mathrm{~mm}\right)$.

\begin{tabular}{|c|c|c|c|c|c|c|c|}
\hline$w$ & $h$ & $t_{1}$ & $l_{1}$ & $t_{2}$ & $l_{2}$ & $t_{3}$ & $l_{3}$ \\
\hline \hline 2.7 & 3.506 & 0.25 & 0.901 & 6.61 & 1.435 & 7.065 & 1.433 \\
\hline
\end{tabular}

terrestrial applications. Figure 7 shows the geometrical parameters of the symmetrical filter under consideration. The structure is implemented in a hollow and uniform below cut-off rectangular waveguide commonly referred as housing $\left(a_{h} \times b_{h}\right)$, which is ended with standard WR-90 waveguide access ports above cut-off. The ridges are centered and have the same width $w$ and height $h$; all the dimensions are summarized in Table I.

Figure 8(a) shows the magnitude of the scattering parameters for the evanescent mode filter. The passband response was designed to be centered at $10 \mathrm{GHz}$ with $300 \mathrm{MHz}$ of bandwidth. We have simulated the structure considering lossless walls and also with a finite conductivity value of $\sigma=5.8 \cdot 10^{7} \mathrm{~S} / \mathrm{m}$. In this second case we included the propagation losses using both the classical power-loss and the proposed method. A frequency shift of about $10 \mathrm{MHz}$ is observed in the response with finite conductivity. HFSS results confirm the downwards frequency shift, although the ripple of the $S_{11}$ parameter has not reached full convergence after 35 iterations. This shift, 


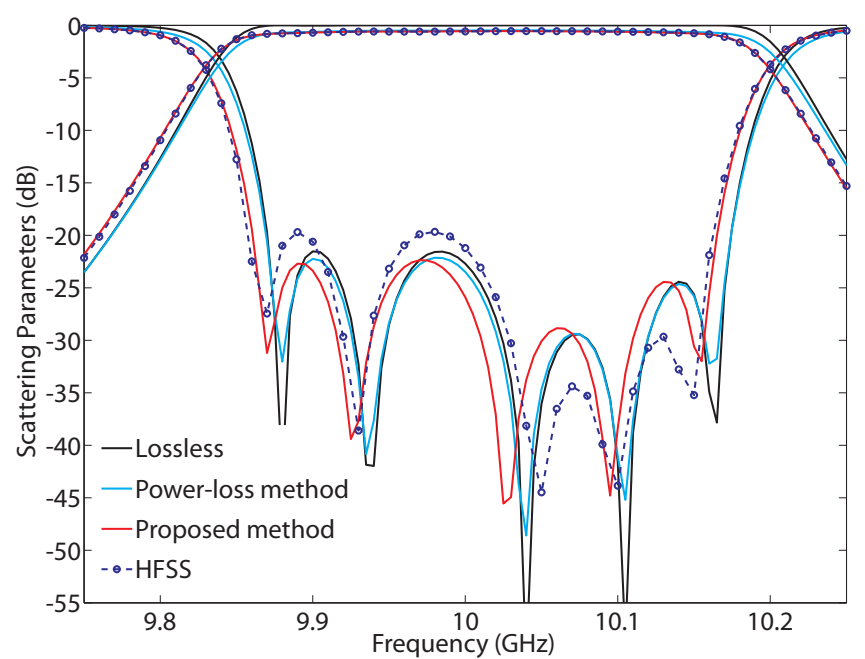

(a)

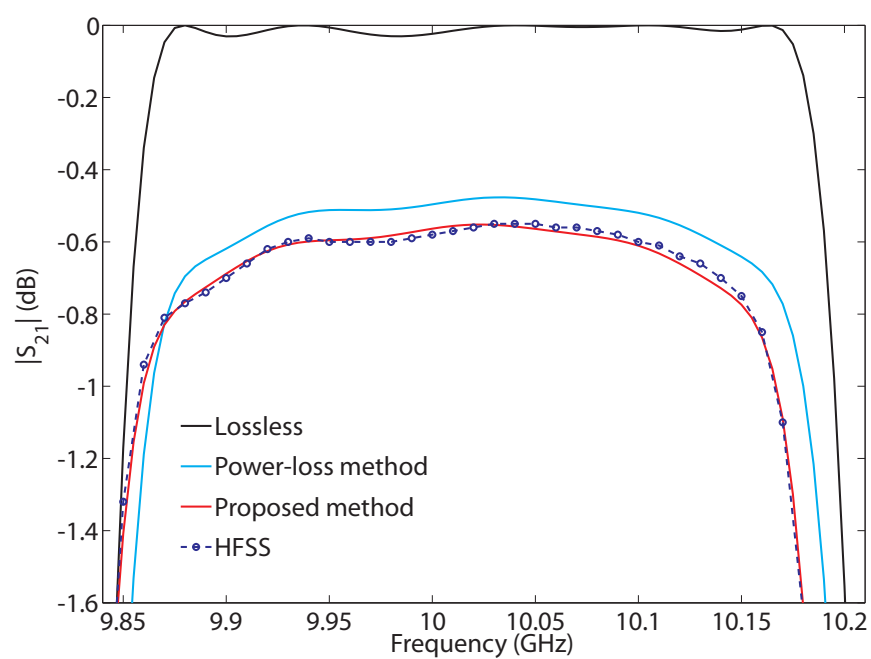

(b)

Fig. 8. In a) magnitude of the scattering parameters of the five order evanescent mode ridge waveguide filter described in Fig. 7 and Table I. In b) detailed view of the magnitude of the transmission coefficient $\left(S_{21}\right)$ in the passband for the same filter.

caused by a variation of the value of the phase constant $\beta$, is not predicted by the power-loss method.

Furthermore, a different minimum insertion loss level (see Fig.8(b)) is obtained by the power-loss method (0.46 $\mathrm{dB})$, HFSS $(0.55 \mathrm{~dB})$ and the proposed technique $(0.54 \mathrm{~dB})$. Note that the rectangular housing is under cut-off, so its losses are not taken into account by the traditional power-loss method. For comparison, such results were obtained using 50 accessible modes, 300 basis functions, and 700 kernel terms in the in the analysis of each discontinuity. These simulating parameters involved a total CPU effort of 14 minutes (201 frequency points) on the Intel Core i7-970 platform at $3.2 \mathrm{GHz}$ with 12-GB RAM, while HFSS simulation takes close to 10 hours, which is approximatively forty times slower than using our software.

Next, the theory developed in Sections II and III has been used to analyze the propagation loss effects of a dual-mode filter with circular cavities for narrow Ka-band applications. The four-pole filter, centered at $30 \mathrm{GHz}$ and with $120 \mathrm{MHz}$ of bandwidth, has been designed using the FEST3D synthesis tool [33]. In the proposed filter topology, the circular cavities are coupled through a cross iris, and are connected with input and output WR-28 port waveguides $(7.112 \times 3.556 \mathrm{~mm})$ by means of rectangular irises; additionally, coupling and tuning screws with rectangular cross-section are placed inside each cavity. The coupling screws are placed, respectively, at 45 and 135 degrees with respect to the $x$-axis. In order to independently adjust each resonance in the same circular cavity, the tuning screws are set in the horizontal and vertical axes. The dimensions of the input/output rectangular irises are $4.118 \times 1.0 \mathrm{~mm}$, the radius of both circular cavities is $4.6 \mathrm{~mm}$ and their length is $9.025 \mathrm{~mm}$; the horizontal arm of the cross iris has dimensions $2.965 \times 0.5 \mathrm{~mm}$ and the vertical arm $0.5 \times 3.516 \mathrm{~mm}$; the thickness of all irises is set to $1 \mathrm{~mm}$. Finally, the width of the screws is $1 \mathrm{~mm}$ and their penetration depths in the cavities are $1.046,0.4$ 


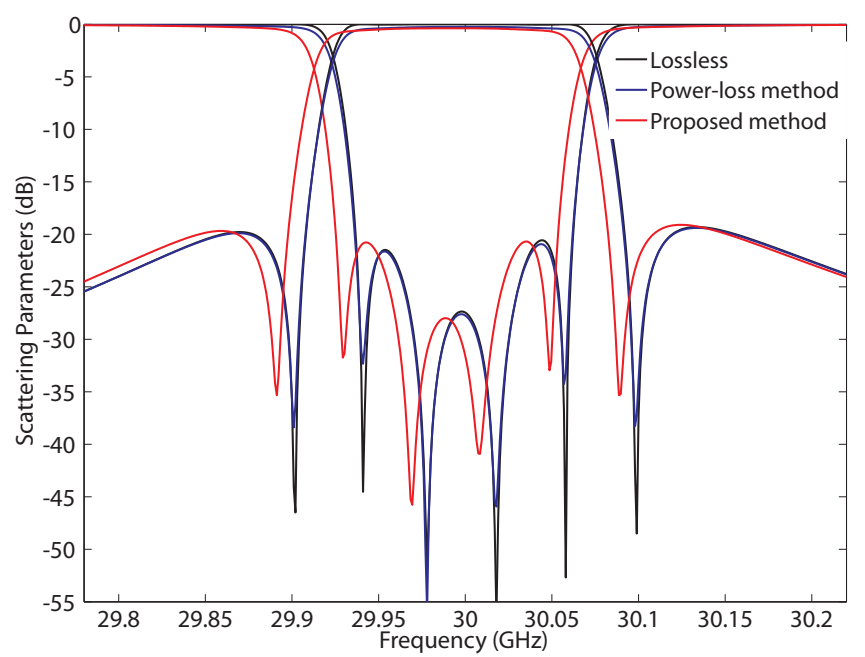

(a)

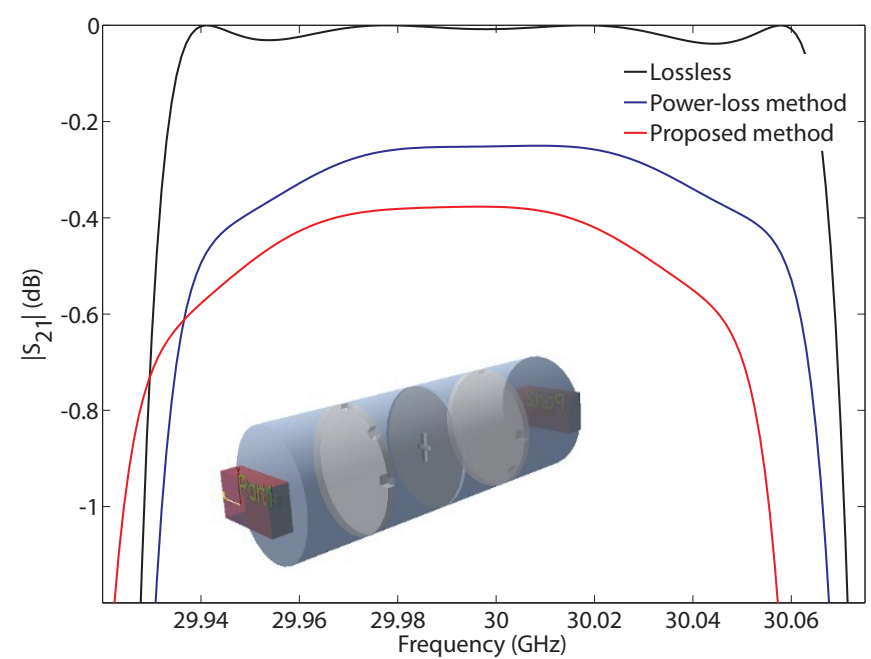

(b)

Fig. 9. In a) magnitude of the scattering parameters of a four order dual-mode filter with circular cavities; in b) detailed view of the magnitude of the transmission coefficient $\left(S_{21}\right)$ in the passband.

and $0.744 \mathrm{~mm}$, respectively, for the horizontal, vertical and diagonal one.

In Fig. 9(a) we compare the magnitude of the scattering parameters of this filter considering the lossless case and a structure made of aluminum with a finite conductivity value of $\sigma=3 \cdot 10^{7} \mathrm{~S} / \mathrm{m}$; in this second case we show the results obtained with both methods. As in the previous case, we can find relevant differences in the results: again a noticeable downwards frequency shift of $11 \mathrm{MHz}$ in the electrical response ( $9 \%$ of the filter bandwidth) is predicted when we use the perturbation of boundary conditions technique, and a higher minimum insertion loss level (see Fig.9(b)) is obtained with respect to the traditional power-loss method.

Finally, in order to check the validity of the software tool with real measurements, the twist component for K-band application originally designed in [26] has also been analyzed. The structure presents a compact geometry based on a soft rotation of the $E$-field through successive square, circular and elliptical waveguides. A detailed view of the internal pieces of the device is shown in Fig. 10; the input/output ports are standard WR-34 rectangular waveguides $(8.636 \times 4.318 \mathrm{~mm})$; the two square waveguides have a side of $8.636 \mathrm{~mm}$ and length of $1.8 \mathrm{~mm}$; the central piece is composed of two circular waveguides of radius $6.1 \mathrm{~mm}$ and length $1.60 \mathrm{~mm}$, and an inner elliptical iris of length $0.30 \mathrm{~mm}$ with major semiaxis of $6.0 \mathrm{~mm}$, minor semiaxis of $3.9 \mathrm{~mm}$, and a rotation of $45^{\circ}$ degrees with respect to the $x$-axis. The measurements of a prototype of such device, operating at $26.3 \mathrm{GHz}$ and with a bandwidth of approximately $2 \mathrm{GHz}$, have been completely recovered by simulated data using a conductivity value of $\sigma=10^{6} \mathrm{~S} / \mathrm{m}$. The frequency shift with respect to the lossless case is negligible in this structure, since ohmic losses are very small (due to the absence of resonances and the $2 \mathrm{GHz}$ bandwidth). The authors have also verified that the frequency shift increases with insertion loss, and therefore is more relevant in narrow-band and sensitive 


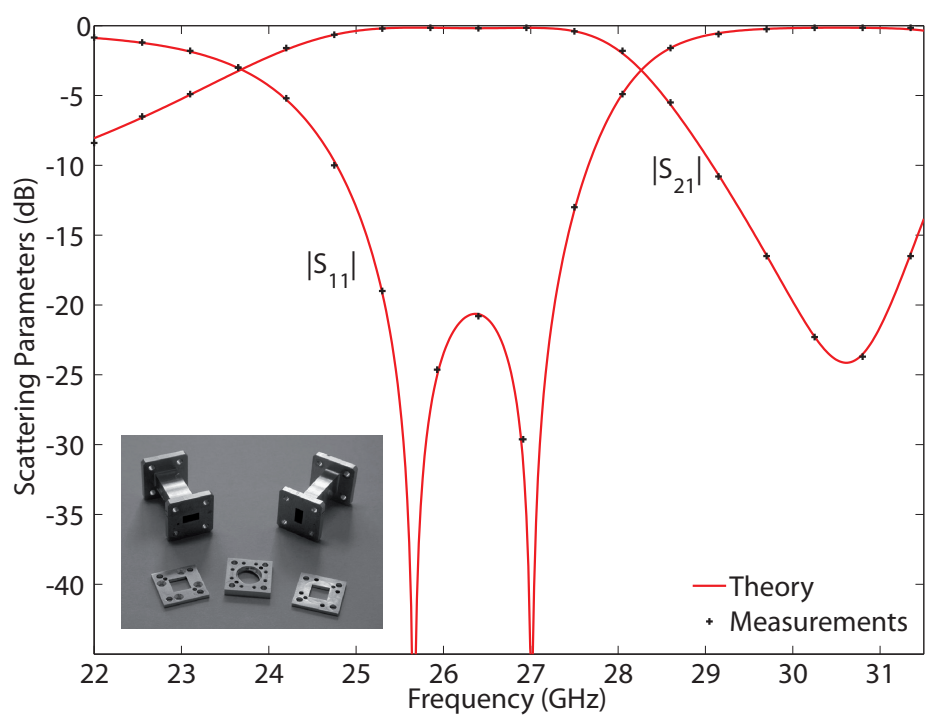

Fig. 10. Comparison between simulated and measured scattering parameters for the manufactured 90-twist component. To recover the measurements, a finite conductivity value of $\sigma=10^{6} \mathrm{~S} / \mathrm{m}$ has been assumed in the simulation.

devices operating at high frequency bands.

\section{Conclusions}

This paper opens the application range of the perturbation of boundary conditions method to waveguides whose contour is composed of any combination of straight, circular and elliptical arcs. As a result, the rigorous evaluation of propagation losses in structures based on arbitrarily shaped waveguides can be carried out, overcoming the limitations of the classic power-loss method: computation of the attenuation constant in the vicinity of the cut-off frequency, losses associated with the evanescent modes, and effect of losses on the phase constant. The new modal analysis tool has been first successfully verified through three examples of well-known waveguides: a doubleridge waveguide, an elliptical waveguide and a circular waveguide, and also applied to a sectorial shaped waveguide and a triangular waveguide. Then, such tool has been integrated into a CAD software package for the advanced prediction of the propagation loss effects in complex waveguide components. The resulting full-wave analysis tool has revealed to be around forty times faster than the well-known finite element commercial software widely used to evaluate component losses. In addition, comparative benchmarks demonstrate that the proposed technique predicts a downwards frequency shift in the electrical response of waveguide devices that can be critical for sensitive narrow-band applications. This frequency shift, caused by a variation of the value of the phase constant $\beta$ due to the ohmic losses, cannot be predicted by the traditional power-loss method. 


\section{ACKNOWLEDGMENTS}

This work has been supported by the Ministerio de Economía y Competitividad (MINECO), Spanish Government, by the coordinated R\&D project TEC2010-21520-C04 and the Grant JC2009-0221, and by University of Alicante under the project GRE10 - 22 .

\section{APPENDIX A}

\section{ANALYTICAL SOLUTION FOR THE SINGULAR INTEGRAL}

This appendix includes the analytical solution of the singular integral emerging in the evaluation of the contour integrals which involves the transverse gradient $\nabla_{t} g$

$$
\begin{aligned}
I_{s}\left(\xi, a_{2}, a_{1}, a_{0}\right) & =\int_{-0.5}^{0.5} \frac{a_{2} \xi^{2}+a_{1} \xi^{\prime}+a_{0}}{\xi-\xi^{\prime}} d \xi^{\prime} \\
& =\int_{\xi-0.5}^{\xi+0.5} \frac{a_{2}(\xi-u)^{2}+a_{1}(\xi-u)+a_{0}}{u} d u \\
& =\left(a_{2} \xi+a_{1}\right)[\xi(\log |\xi+0.5|-\log |\xi-0.5|)-1] \\
& +a_{0}[\log |\xi+0.5|-\log |\xi-0.5|]
\end{aligned}
$$

\section{REFERENCES}

[1] Rogers, D. V., Ippolito, L. J., and Davarian, F.: System requirements for Ka-band earth-satellite propagation data, Proceedings of the IEEE, 1997, 85, (5), pp. 810-820.

[2] Cazaux, J. L., Cayrou, J. C., Miquel, C., et al.: New generation of Ka-band equipment for telecommunication satellites. Proc. of 34th European Microwave Conference, Amsterdam, Oct. 2004, pp. 325-328.

[3] Yu, M., Yassini, B., Panariello, A., and Healy, G.: Passive components for advanced satellite systems. Proc. of IEEE MTT-S Int. Microw. Symp. Dig, Montreal, Canada, Jun. 2012, pp. 1-3.

[4] Gopal, R., and Fang, R. J.: High-capacity Ka commercial communications satellite architectures for defense applications. Proc. of MILCOM Military Communications Conf., Orlando, Fl., Oct. 2012, pp. 1-6.

[5] Daniels, R. C., Murdock, J. N., Rappaport, T. S. and Heath, R. W.: 60 GHz Wireless: up close and personal, IEEE Microwave Magazine, 2010, 11, (7), pp. 44-50.

[6] Park, C., and Rappaport, T. S.: Short-range wireless communications for next-generation networks: UWB, 60 GHz millimeter-wave WPAN, and zigBee, IEEE Wireless Communications, 2007, 14, (4), pp. 70-78.

[7] Ettorre, M., Sauleau, R., Le Coq, L., and Bodereau, F.: Single-folded leaky-wave antennas for automotive radars at 77 GHz, IEEE Antennas and Wireless Propagation Letters, 2010, 9, pp. 859-862. 
[8] Uher, J., Bornemann, J., and Rosemberg, U.: Waveguide components for antenna feed systems:theory and CAD (Artech House, Boston-London, 1993).

[9] Hunter, I., Bilonet, L., Jarry, B., and Guillon, P.: Microwave filter- applications and technology, IEEE Trans. Microw. Theory Tech., 2002, 50, (3), pp. 794-805.

[10] Guyette, A. C., Hunter, I. C., and Pollard, R. D.: The design of microwave bandpass filters using resonators with nonuniform Q, IEEE Trans. Microw. Theory Tech., 2006, 54, (11), pp. 3914-3922.

[11] Miraftab, V., and Yu, M.: Generalized lossy microwave filter coupling matrix synthesis and design using mixed technologies, IEEE Trans. Microw. Theory Tech., 2008, 56, (12), pp. 3016-3027.

[12] Oldoni, M., Macchiarella, G., Gentili, G. G., and Ernst, C.: A new approach to the synthesis of microwave lossy filters, IEEE Trans. Microw. Theory Tech., 2010, 58, (5), pp. 1222-1229.

[13] Szydlowski, L., Lamecki, A., and Mrozowski, M.: Design of microwave lossy filter based on Substrate Integrated Waveguide (SIW), IEEE Microw. Wireless Compon. Lett., 2011, 21, (5), pp. 249-251.

[14] Collin, R. E.: Field Theory of Guided Waves (McGraw-Hill, New York, 1960), ch. 5, pp. 182-183.

[15] Rong, Y., and Zaki, K. A.: Characteristics of generalized rectangular and circular ridge waveguides, IEEE Trans. Microw. Theory Tech., 2000, 48, (2), pp. 258-265.

[16] Leal-Sevillano, C. A., Ruiz-Cruz, J. A., Montejo-Garai, J. R., and Rebollar, J. M.: Field propagation in circular hollow waveguides with non-ideal metallic conductors from microwaves to terahertz frequencies, IEEE Trans. Microw. Theory Tech., 2011, 59, (12), pp. 3013-3022.

[17] Che, W.-Q., Li, C.-X., Wang, D.-P., Xu, I., and Chou, Y. L.: Investigation on the ohmic conductor losses in substrate-integrated waveguide and equivalent rectangular waveguide, Journal of Electromagn. Waves and Appl., 2007, 21, (6), pp. 769-780.

[18] Nikolski, V. V.: Electrodynamic and Radiowave Propagation (MIR, Moscow, 1973).

[19] Jackson, J. D.: Classical Electrodynamics (John Wiley and Sons, New York, 1999), ch. 8, pp. 363-368.

[20] Marini, S., Mattes, M., Gimeno, B., and Boria, V. E.: Improved computation of propagation losses in waveguide structures using perturbation of boundary conditions, IEEE Microw. Wireless Compon. Lett., 2011, 21, (11), pp. 577-579.

[21] Marini, S., Mattes, M., Gimeno, B., Soto, P., and Boria, V. E.: Advanced analysis of propagation losses in rectangular waveguide structures using perturbation of boundary conditions. Proc. of IEEE MTT-S Int. Microwave Workshop series on Millimeter Wave Integr. Tech., Sitges, Spain, Sep. 2011, pp. 680-683.

[22] Jiao, C.-Q.: Selective suppression of electromagnetic modes in a rectangular waveguide by using distributed wall losses, Progress in Electromagnetics Research Letters, 2011, 22, pp. 119-128.

[23] Yan, S., Huang, B. K., Jiang, W. S., and Jiang, Y. S.: Calculation of the propagation constants in waveguides with imperfect 
conductor by perturbed boundary condition method, Journal of Microwaves, 2010, 26, (2), pp. 35-38 (in Chinese).

[24] Hung, C.-L., and Yeh, Y. S.: The propagation constants of higher-order modes in coaxial waveguides with finite conductivity, Int. J. Infrared and Millimeter Waves, 2005, 26, (1), pp. 29-39.

[25] Conciauro, G., Bressan, M. and Zuffada, C.: Waveguide modes via an integral equation leading to a linear matrix eigenvalue problem, IEEE Trans. Microwave Theory Tech., 1984, 32, (11), pp. 1495-1504.

[26] Cogollos, S., Marini, S., Boria, V. E., et al.: Efficient modal analysis of arbitrarily shaped waveguides composed of linear, circular and elliptical arcs using the BI-RME method, IEEE Trans. Microwave Theory and Tech., 2003, 51, (12), pp. 2378-2390.

[27] Gerini, G., Guglielmi, M., and Lastoria, G.: Efficient integral equation formulations for admittance or impedance representation of planar waveguide junctions. Proc. of IEEE MTT-S Int. Microw. Symp. Dig., Honolulu, HI, Jun. 1998, pp. 1747-1750.

[28] Conciauro, G., Guglielmi, M., and Sorrentino, R.: Advanced Modal Analysis (John Wiley \& Sons, New York, 2000).

[29] HFSS, ANSYS Inc., Southpointe 275 Technology Drive Canonsburg, PA 15317.

[30] Elsherbeni, A., Kaifez, D., and Zeng S.: Circular sectorial waveguides, IEEE Antennas and Propagation Mag., 1991, 33, (6), pp. 20-27.

[31] Overfelt, P. L., and White D. J.: TE and TM modes of some triangular cross-Section waveguides uising superposition of plane waves, IEEE Trans. Microwave Theory and Tech., 1986, 34, (1), pp. 161-167.

[32] Soto, P., De Llanos, D., Boria, V. E., et al.: Performance analysis and comparison of symmetrical and asymmetrical configurations of evanescent mode ridge waveguide filters, Radio Science, 2009, 44, RS6010.

[33] FEST3D 6.7, Aurora Software and Testing S.L. (on behalf of ESA/ESTEC), Valencia, Spain. http//www.fest3d.com, accessed June 2013. 